\title{
Colonoscopy performance is stable during the course of an extended three-session working day
}

Authors

Institutions

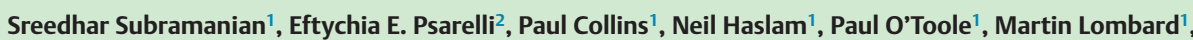
Sanchoy Sarkar ${ }^{1}$

${ }^{1}$ Department of Gastroenterology, Royal Liverpool University Hospital, United Kingdom

${ }^{2}$ Cancer Research UK Liverpool Cancer Trials Unit, Liverpool, United Kingdom submitted

30. November 2014 accepted after revision 6. May 2015

\section{Bibliography}

DOI http://dx.doi.org/

10.1055/s-0034-1392523

Published online: 11.8.2015

Endoscopy International Open

2015; 03: E494-E500

(C) Georg Thieme Verlag KG

Stuttgart - New York

E-ISSN 2196-9736

\section{Corresponding author}

\section{Sreedhar Subramanian, MD,} MRCP

Department of

Gastroenterology

Royal Liverpool University

Hospital

Prescot Street

Liverpool L7 8XP

United Kingdom

Fax: +44-151-7065832

sreedhar.subramanian@rlbuht. nhs.uk

\section{License terms}

()(1) $\Theta \circledast$
Background: Three-session days were introduced in our endoscopy unit to accommodate the increased demand resulting from the introduction of the National Health Service Bowel Cancer Screening Programme (BCSP). Cecal intubation rate (CIR) and adenoma detection rate (ADR) may decline with time during a standard working day, but data are lacking for an extended three-session day. We assessed colonoscopy performance in an extended three-session day.

Methods: Colonoscopies performed during the year 2011 were retrospectively analyzed. The CIR and ADR were analyzed according to the time of day when procedures were done: morning (AM), afternoon (PM), or evening (EVE). Because of an expected higher incidence of adenomas in the BCSP patients, ADR was analyzed according to indication (BCSP or non-BCSP).

Results: Of the 2574 colonoscopies, 1328 (51.7\%) were in male patients and 1239 (48.3\%) in female

\section{Introduction}

\section{$\nabla$}

Globally, the demand for gastrointestinal endoscopic procedures is increasing. The department of health in the United Kingdom has predicted that there will be a $10 \%$ to $15 \%$ year-on-year demand for endoscopic procedures [1]. This equates to approximately 220 additional endoscopy sessions for a large hospital [1]. The increased demand is partly driven by the provision of the Bowel Cancer Screening Programme (BCSP), which was introduced in 2006, but other factors, such as a lower threshold for endoscopy, also play a role. In order to cope with this increased demand, some endoscopy units have introduced a three-session working day.

Several studies have suggested that time of day may affect colonoscopy outcomes, possibly as a consequence of endoscopist fatigue. The cecal intubation rate (CIR) has been reported to be higher in morning lists than in afternoon lists [2,3], but patients with a median age of 63 years (interquartile range [IQR], $51-70)$. Of the 2574 colonoscopies, 1091 (42.4\%) were performed in AM lists, 994 (38.6\%) in PM lists, and 489 (19\%) in EVE lists. Time of day did not affect the CIRs for the AM, PM, and EVE lists $(90.5 \%, 90.1 \%$, and $89.9 \%$, respectively; $\left.x^{2}[2, \mathrm{~N}=2540]=0.15, P=0.927\right)$. The CIR was reduced in female patients and those with poor bowel preparation $(P<0.05)$. After exclusion of the BCSP patients, the ADR was lower in the EVE lists than in the AM and PM lists on univariate analysis, but on multivariate analysis, this difference was not significant $(P>0.05)$. The ADR was significantly higher in patients older than 60 years and in men $(P<0.001)$. Queue position did not independently influence the CIR or ADR.

Conclusions: Colonoscopy quality does not appear to depend on time of day or queue position in an extended three-session day.

this difference is not adequately explained by differences in bowel preparation [3]. Similarly, the adenoma detection rate (ADR), another quality indicator of colonoscopy, has been reported to decline as the day progresses. For instance, Chan et al. reported that more adenomas were detected in morning lists than in afternoon lists [2], an observation replicated by others [4]. However, this is not a consistent finding, and other groups have reported that the ADR remains stable throughout the day $[5,6]$.

Akin to time of day, queue position is another variable that is known to influence the polyp detection rate (PDR). Lee et al. reported a $4.6 \%$ reduction in the PDR with each elapsed hour in the day [7], and a similar finding of a time-related decline in the ADR was reported by another study [2]. A study from Mayo Clinic investigated the effect on the PDR of a three-session day with 3-hour shift schedules [8]. The authors found that the PDR varied significantly by shift, with more 
polyps detected in shift 2 (1030-1330 hours) than in shift 1 (0730-1030 hours) and shift 3 (1330-1630 hours). However, only a single study has examined the effect of extending the working day on indicators of colonoscopy quality [9]. In a study from the United Kingdom, Thurtle et al. reported that the PDR remained stable throughout the day, including evening sessions [9]. In this study, we further examined the effect of a 12-hour work schedule on colonoscopy outcomes.

\section{Methods}

This retrospective study was conducted at the Royal Liverpool University Hospital, a tertiary hospital in the United Kingdom. Additional evening lists were introduced in our unit in 2008, and the study included procedures conducted in 2011.During the study period, there were four morning (AM) endoscopy lists (0830-1230 hours), four afternoon (PM) lists (1230-1630 hours), and one or two evening (EVE) lists (1630-2000 hours) on Mondays through Thursdays, with only AM and PM lists scheduled on Fridays. Subjects with diabetes were placed on AM or PM lists, not on EVE lists. Endoscopists typically operated on a shift basis with 4-hour blocks, but some endoscopists performed back-to-back lists (AM and PM or PM and EVE), with a short break in between. Before a PM or EVE endoscopy list, operators typically had other commitments, such as outpatient clinics or inpatient consults.

All procedures were conducted by an experienced endoscopist or by a trainee under the direct supervision of an experienced endoscopist. An experienced endoscopist was defined as an operator who had completed fellowship training and had been accredited for independent colonoscopy practice by the Joint Association for Gastroenterology (JAG). Scheduled endoscopy lists for each day between January 1 and December 31, 2011, were obtained from an electronic database. We excluded lists containing only esophagogastroduodenoscopy (EGD), flexible sigmoidoscopy, or endoscopic ultrasound procedures. Lists containing at least two colonoscopies were included in the study. We chose an arbitrary cutoff of two colonoscopies in order to determine the effect of repetitive fatigue on colonoscopy outcome. We also excluded patients with incomplete data.

We performed a retrospective chart review study of all eligible patients. The following data were abstracted: date and time of colonoscopy, patient gender and age, number of patients on each endoscopy list, number of colonoscopies on each list, colonoscopy queue position, presence of a trainee, sedation used, American Society of Anesthesiologists (ASA) status of the patient, indication for the procedure, quality of the bowel preparation, operator experience, cecal intubation status, number of polyps detected, number of polyps removed, and subsequent histologic findings. Subjects were classified into two age groups: younger than 60 years and 60 years or older. Colonoscopy withdrawal time is not routinely recorded in our institute and was not available for analysis.

Bowel preparation was achieved with either sodium picosulphate (Picolax; Ferring Pharmaceuticals) or polyethylene glycol (MoviPrep; Valeant Pharmaceuticals International). Patients scheduled for the AM list took their bowel preparation the previous day, patients scheduled for the PM list took a split-dose bowel preparation, and EVE patients took all of their preparation on the same day. Endoscopists graded the quality of bowel preparation at the time of the procedure on a previously specified scale of poor (so- lid or adherent stool impossible to wash away with no exclusion value), satisfactory (opaque fluid and some adherent stool that could be washed away to provide reasonable views for exclusion), or good (clear fluid only or opaque fluid that was easily suctioned), as described previously [10].

The time of colonoscopy was stratified into three categories according to the start time of the scheduled list: AM, PM, or EVE. Queue position was defined as the number of colonoscopies performed during a particular session and was categorized as position $1,2,3$, or 4 or higher. We assigned a new queue position for each session because many endoscopists worked for just a halfday block, and the new operator for the afternoon would start with a new "fatigue clock." The majority of procedures were performed with the patient under conscious sedation, achieved with the administration of a combination of intravenous meperidine (pethidine) or fentanyl along with midazolam. Olympus (Tokyo, Japan) colonoscopes of variable stiffness were used for the procedure.

The CIR was defined as the percentage of colonoscopies in which the cecum was intubated as recorded by the endoscopist. Identification of the ileocecal valve, appendiceal orifice, and triradiate cecal fold or ileal intubation was taken as confirmation of cecal intubation. The ADR was defined as the percentage of colonoscopies in which at least one adenoma was detected per colonoscopy. Adenomas were defined as polyps with adenomatous tissue, including sessile serrated adenomas and advanced adenomas confirmed by pathology.

The CIR and ADR were analyzed according to the session in which the patient was scheduled for the procedure (AM, PM, or EVE). Our initial analysis included all patients included in the original data set. We subsequently analyzed procedures done as part of the BCSP separately. Operators in the BCSP should have performed at least 1000 colonoscopies in their career with a CIR above $90 \%$ and an ADR above $20 \%$ in the preceding 12 months. They also undertake a summative assessment of knowledge and skills to test their competency. The patients who have a colonoscopy as part of the BCSP have a moderate risk for colorectal cancer as indicated by a positive fecal occult blood test. In addition, BCSP patients are typically scheduled for an AM or PM list, not an EVE list. In order to exclude confounding from these factors, we analyzed the ADR with and without BCSP patients. The study was approved by the clinical audit department of Royal Liverpool University Hospital.

\section{Statistical analysis}

Descriptive statistics were computed for the entire study population for all predictor variables. Categorical variables are presented as proportions and were compared with Fisher's exact test or the $\mathrm{X}^{2}$ test. Continuous variables that were not normally distributed are presented with their medians and IQR and were compared with the Kruskal-Wallis test. Multivariate logistic regression analyses were performed in order to allow for confounding between potential patient- and endoscopy-related variables. Odds ratios (ORs) and 95\% confidence intervals (CIs) were calculated. The following variables were tested: time of day, age, queue position, gender, trainee presence, experience, and quality of bowel preparation, as well as the two-way interaction terms between the predictor variables. The Akaike Information Criterion (AIC) was used to select the final multivariate models, in which only variables achieving in univariate analyses $P$ values of less than 0.20 were included. As our data set consisted of different endoscopists, clustered sandwich variance estimators were used in or- 
Total number of colonoscopies in study period: 3370

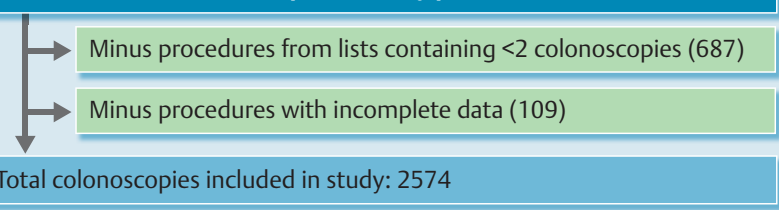

Fig. 1 Flowchart with total number of colonoscopies done in the year 2011 at the Royal Liverpool Hospital endoscopy unit, application of exclusion criteria, and total number of included procedures.

der to adjust for the variability of operators. The Pearson chisquared test was used to ensure adequate fit of the model. Results were declared as significant if a two-sided $P$ value of 0.05 or lower was achieved. All statistical calculations were performed with Stata 13 software (StataCorp. 2013. Stata Statistical Software: Release 13. College Station, Texas: StataCorp LP).

\section{Results \\ $\nabla$}

\section{Baseline characteristics}

Between January and December 2011, a total of 3370 colonoscopies were performed at our institution ( $\bullet$ Fig. 1). A total of 2574 colonoscopies were included in the study, 1328 (51.7\%) in male patients and $1239(48.3 \%)$ in female patients. The median age of the patients was 63 years (IQR $51-70$ ). Of the 2574 colonoscopies, 1091 (42.4\%) were performed in AM lists, 994 (38.6\%) in PM lists, and 489 (19\%) in EVE lists. All the procedures during the study period were performed either directly by or under the supervision of 15 experienced operators. The baseline characteristics of the patients undergoing colonoscopy are shown in $\bullet$ Table 1. The EVE lists had a significantly greater proportion of patients younger than 60 years of age and of ASA category 1 patients $(P<0.001)$. There was no significant difference in the proportion of lists containing three or four colonoscopies across the three sessions $(P=0.585)$. Bowel preparation was noted to be poor in a greater proportion of AM cases (11.9\% of AM cases, $8.8 \%$ of PM cases, and $7.6 \%$ of EVE cases, $P<0.001)$.

Cecal intubation rate remains stable throughout the day The overall unadjusted CIR in our study was $90.24 \%$. This was confirmed by ileal intubation in $30.83 \%$ of cases. There was no significant difference in the unadjusted CIR across the three sessions (90.5\% AM, 90.1\% PM, and 89.9\% EVE; $\mathrm{X}^{2}[2, \mathrm{~N}=2540]=0.15$, $P=0.927$ ( $\odot$ Fig. 2a). On univariate analysis, queue position was not independently associated with $\operatorname{CIR}\left(\mathrm{X}^{2}[3, \mathrm{~N}=2535]=0.53, P=\right.$ 0.53 ; $\odot$ Table $2 \mathrm{a}$ ) despite a numerical increase in cecal intubation for queue positions 3 and 4 ( $\bullet$ Fig. 2 b). On multivariate logistic regression analysis, gender and quality of bowel preparation were found to be associated with cecal intubation, whereas trainee presence was not $(\mathbf{O}$ Table $\mathbf{2} \mathbf{b})$. More specifically, the odds of cecal intubation in female patients were $39 \%$ lower than those for male patients after adjustment for the other covariates in the model (OR 0.61, 95\%CI 0.45-0.83, P=0.002). Quality of bowel preparation significantly affected the CIR; the odds of cecal intubation with satisfactory bowel preparation were 4.4 times greater (OR 4.37, 95\%CI 2.87-6.65, $P<0.001)$ and the odds with good bowel preparation were 4.8 times greater (OR $4.77,95 \% \mathrm{CI} 3.01$ $7.56, P<0.001)$ than the odds with poor bowel preparation after adjustment for gender and trainee presence in the model.

\begin{tabular}{|c|c|c|c|c|c|}
\hline & Total & AM & PM & EVE & Pvalue \\
\hline \multicolumn{6}{|l|}{ Indication for colonoscopy, n (\%) } \\
\hline Diarrhea & $363(14.11)$ & $139(12.75)$ & $148(14.89)$ & $76(15.54)$ & 0.225 \\
\hline Previous polyps & $411(15.97)$ & $164(15.05)$ & $158(15.90)$ & $89(18.20)$ & 0.285 \\
\hline BSCP & $434(16.86)$ & $193(17.69)$ & $235(23.64)$ & $6(1.23)$ & $<0.001$ \\
\hline Gender, $\mathrm{n}(\%)$ & & & & & 0.064 \\
\hline Female & $1239(48.27)$ & $544(50)$ & $449(45.35)$ & $246(50.31)$ & \\
\hline Male & $1328(51.73)$ & $544(50)$ & $541(54.65)$ & $243(49.69)$ & \\
\hline Age (categorical), n (\%) & & & & & $<0.001$ \\
\hline$\geq 60 y$ & $1576(61.23)$ & $725(66.45)$ & $680(68.41)$ & $171(34.97)$ & \\
\hline$<60 y$ & $998(38.77)$ & $366(33.55)$ & $314(31.59)$ & $318(65.03)$ & \\
\hline Age (continuous) & & & & & $<0.001$ \\
\hline Median age $(26 \%-75 \%)$, y & $63(51-70)$ & $65(54-72)$ & $64(54-71)$ & $52(42-63)$ & \\
\hline ASA score, $\mathrm{n}(\%)$ & & & & & $<0.001^{1}$ \\
\hline 1 & $1246(48.46)$ & $456(41.87)$ & $510(51.36)$ & $280(57.26)$ & \\
\hline 2 & $1010(39.28)$ & $496(45.55)$ & $378(38.07)$ & $136(27.81)$ & \\
\hline 3 & $92(3.58)$ & $60(5.51)$ & $25(2.52)$ & $7(1.43)$ & \\
\hline 4 & $5(0.19)$ & $4(0.37)$ & $1(0.10)$ & $0(0.0)$ & \\
\hline Not assessed & $218(8.48)$ & $73(6.70)$ & $79(7.96)$ & $66(13.50)$ & \\
\hline Colonoscopies / list, n (\%) & & & & & 0.585 \\
\hline 3 & $1034(40.60)$ & $430(39.67)$ & $382(39.06)$ & $222(45.77)$ & \\
\hline 4 & $1311(51.47)$ & $548(50.55)$ & $503(51.43)$ & $260(53.61)$ & \\
\hline Bowel preparation, n (\%) & & & & & $<0.001$ \\
\hline Poor & $254(9.89)$ & $130(11.94)$ & $87(8.79)$ & $37(7.57)$ & \\
\hline Satisfactory & $924(35.98)$ & $427(39.21)$ & $344(34.75)$ & $153(31.29)$ & \\
\hline Good & $1390(54.13)$ & $532(48.85)$ & $559(56.46)$ & $299(61.15)$ & \\
\hline Trainee presence, $n$ (\%) & $225(9.66)$ & $122(12.54)$ & $83(9.23)$ & $20(4.39)$ & $<0.001$ \\
\hline
\end{tabular}

AM, morning; PM, evening; EVE, evening; BCSP, Bowel Cancer Screening Programme; ASA, American Society of Anesthesiologists.

${ }^{1}$ Fisher's exact test. 

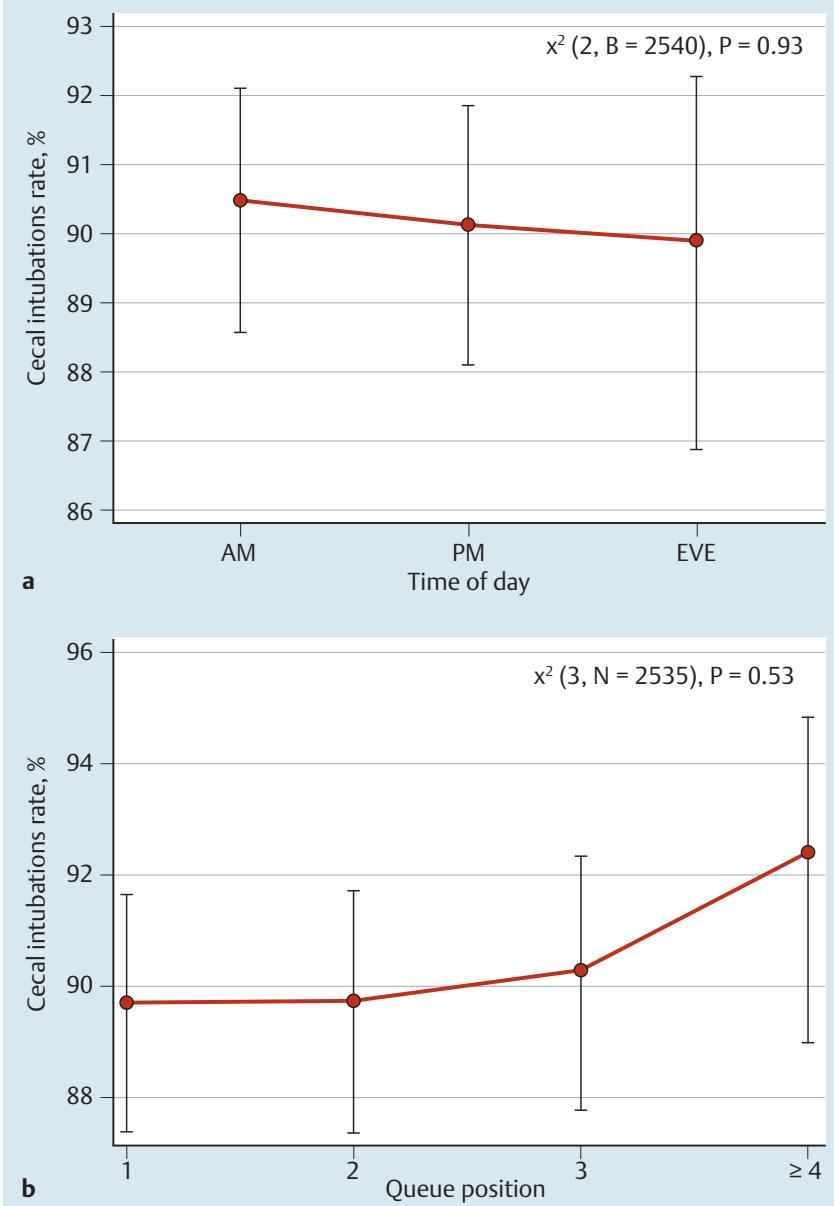

Fig. 2 a Cecal intubation rate by time of day. AM, morning; PM, afternoon; EVE, evening. b Cecal intubation rate by queue position.

\section{Adenoma detection rate}

Overall, adenomas were detected in 702 patients (27.6\%). After exclusion of the BCSP cases, adenomas were detected in 476 cases $(22.5 \%)$. A statistically significant relationship was found between the ADR and time of day (23.6\% AM, 25.7\% PM, and 15.4\% EVE; $X^{2}$ [2, N=2117]=18.827, $P<0.0001$; Fig. 3a), whereas queue position was not associated with the probability of adenoma detection $\left(X^{2}[3, N=2095]=1.80, P=0.615\right.$; $\bullet$ Fig. 3 b and Table $3 \mathbf{a}$ ). A multivariate logistic regression analysis was performed that included the following variables: age, sex, time of day, quality of bowel preparation, and interaction of bowel preparation quality with time of day ( $\bullet$ Table $\mathbf{3 b}$ ). Time of day and quality of bowel preparation were collapsed into two categories in order to allow a direct interpretation of the interaction term. Age, gender, and quality of bowel preparation were significantly associated with adenoma detection. More specifically, the odds of adenoma presence for female patients was decreased by $41 \%$ in comparison with the odds for male patients after adjustment for all other variables in the model (OR 0.59, 95\%CI $0.45-$ $0.76, P<0.001)$. The odds of adenoma presence in patients older than 60 years was 2.4 times greater than that in patients younger than 60 years after adjustment for all the other variables in the model (OR 2.43, 95\%Cl 1.42-3.75, $P=0.001$ ). The odds of adenoma detection were 2.3-fold greater with a satisfactory bowel preparation than with a poor preparation (OR 2.31, 95\%CI 1.31 $3.44, P=0.002)$. Interestingly, the probability of adenoma detec-
Table 2 a Univariate logistic regression analysis of factors associated with cecal intubation.

\begin{tabular}{|llll|}
\hline Variable & Odds ratio & $\mathbf{9 5} \% \mathbf{C l}$ & Pvalue \\
\hline Time & & & \\
\hline PM vs. AM & 0.99 & $0.69-1.42$ & 0.963 \\
\hline EVE vs. AM & 1.27 & $0.88-1.84$ & 0.199 \\
\hline EVE vs. PM & 1.28 & $0.86-1.90$ & 0.219 \\
\hline Age $>60$ vs. $<60$ y & 0.57 & $0.38-0.86$ & 0.007 \\
\hline Queue position ${ }^{1}$ & & & \\
\hline 2 vs. 1 & 1.15 & $0.77-1.70$ & 0.489 \\
\hline 3 vs. 1 & 1.30 & $0.88-1.92$ & 0.184 \\
\hline 4 vs. 1 & 1.42 & $0.84-2.41$ & 0.191 \\
\hline 3 vs. 2 & 1.13 & $0.78-1.64$ & 0.506 \\
\hline 4 vs. 2 & 1.24 & $0.73-2.10$ & 0.431 \\
\hline 4 vs. 3 & 1.09 & $0.68-1.74$ & 0.714 \\
\hline 4 vs. 1, 2, 3 & 1.26 & $0.79-2.00$ & 0.331 \\
\hline Gender: female vs. male & 0.66 & $0.46-0.94$ & 0.023 \\
\hline Bowel preparation & & & \\
\hline Satisfactory vs. poor & 3.63 & $2.18-6.03$ & 0.000 \\
\hline Good vs. poor & 4.79 & $2.90-7.91$ & 0.000 \\
\hline Satisfactory vs. good & 1.32 & $0.91-1.91$ & 0.141 \\
\hline Trainee presence & 0.73 & $0.43-1.23$ & 0.235 \\
\hline Experience & 1.02 & $0.67-1.53$ & 0.934 \\
\hline
\end{tabular}

$\mathrm{Cl}$, confidence interval; PM, afternoon; AM, morning; EVE, evening.

${ }^{1}$ Queue variable was divided into four categories; the fourth category consisted of queues $\geq 4$; age was divided into two groups for data-fitting purposes.

Table 2 b Multivariate logistic regression of cecal intubation, including gender, quality of bowel preparation, and presence of trainee.

\begin{tabular}{|llll|}
\hline Variable & Odds ratio & $\mathbf{9 5 \%} \mathbf{C l}$ & $\boldsymbol{P}$ value \\
\hline Gender: female vs. male & 0.61 & $0.45-0.83$ & 0.002 \\
\hline Bowel preparation & & & \\
\hline Satisfactory vs. poor & 4.37 & $2.87-6.65$ & 0.000 \\
\hline Good vs. poor & 4.77 & $3.01-7.56$ & 0.000 \\
\hline Satisfactory vs. good & 1.09 & $0.66-1.82$ & 0.734 \\
\hline Trainee presence & 0.63 & $2.00-6.17$ & 0.111 \\
\hline
\end{tabular}

$\mathrm{Cl}$, confidence interval.

tion increased with improved quality of bowel preparation for the AM and PM sessions, but the probability of adenoma detection for the EVE session was not affected by the quality of bowel preparation.

\section{Discussion}

In this study, we report that colonoscopy performance remains stable over an extended 12-hour workday schedule. Only two previous studies looked at colonoscopy performance in a threesession day $[8,9]$. Importantly, our observations are similar to those of the recent study by Thurtle et al. [9] but extend their observations to include ADR as a measure of colonoscopy quality. The unadjusted CIR in our study achieved the British Society of Gastroenterology (BSG) target of 90\% [11] with the exception of the EVE list, which was just below the target at $89.9 \%$. The unadjusted CIR in our study is lower than that reported from a recent United Kingdom-wide audit [12]. The data from our study were from an entire year, as opposed to a representative 2-week period in the study by Gavin et al. [12], which could explain the difference between the CIRs in the two studies. Furthermore, inpatients were not excluded from our analysis. Previous studies have 

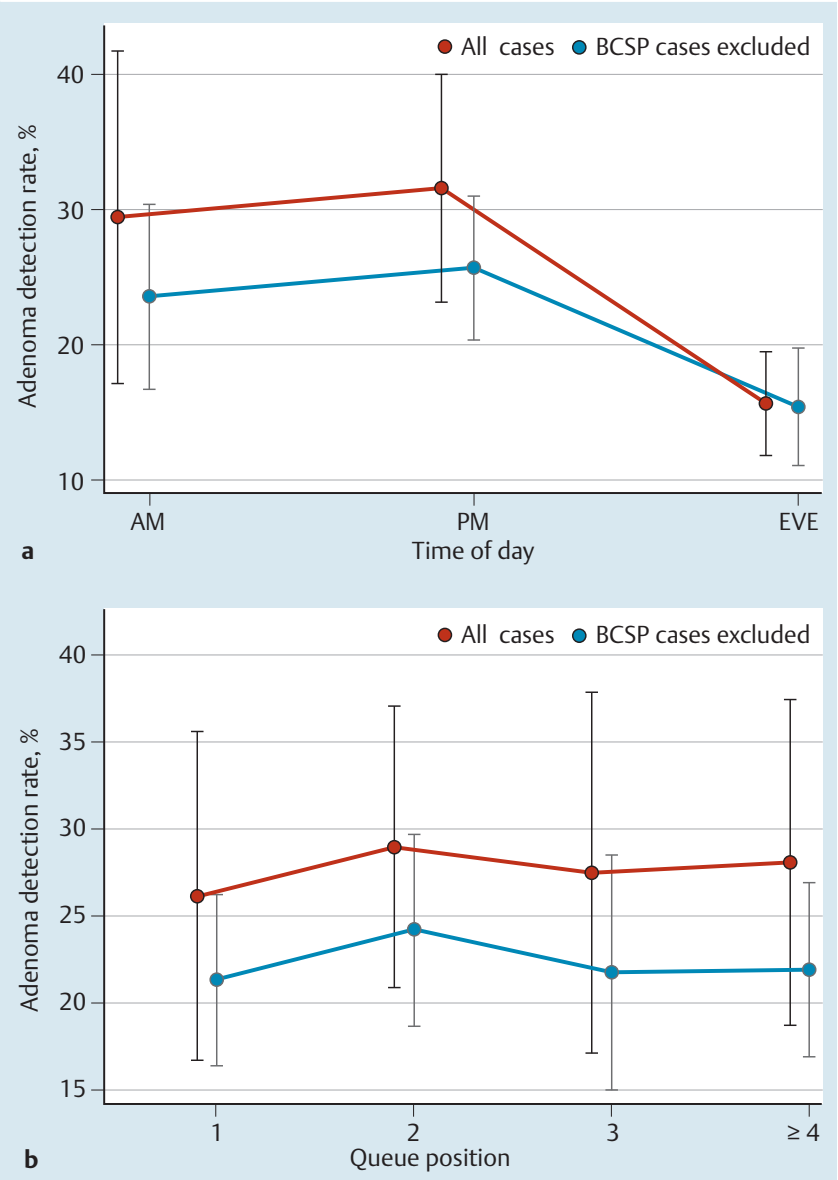

Fig. 3 a Adenoma detection rate by time of day with inclusion of all cases $(n=2574)$ and with exclusion of Bowel Cancer Screening Programme (BCSP) cases $(n=2117)$. b Adenoma detection rate by queue position with inclusion of all cases $(n=2574)$ and with exclusion of BCSP cases $(n=2117)$. AM, morning; PM, afternoon; EVE, evening.

reported lower CIRs among hospitalized patients, caused by such factors as patient immobility, constipation, and poor quality of the preparation $[13,14]$.

Reassuringly, the CIR in our study remained stable throughout the day. Our findings are consistent with those of a previously reported study showing no difference between the CIRs of AM and PM lists [15]. However, other groups have reported a decline in the CIR with increasing time of day and increasing queue position. For instance, studies by Sanaka et al. [3] and Chan et al. [2] independently reported a decline in the CIR with colonoscopies done later in the day. Harewood et al. also reported that the CIR declined with increasing queue position [5]. It is likely that clinical variables influencing colonoscopy completion could account for these differences. We did not record other clinically relevant variables that are known to affect colonoscopy completion rates, such as body mass index (BMI) and previous pelvic surgery [14]. The overall ADR in our study (27.6\%) is similar to that reported in the literature $[3,4,16,17]$ and above that recommended for an unselected colonoscopy population [17]. In our study, the ADR was lower in the EVE lists than in the AM and PM lists in univariate analysis. Several factors could account for a lower ADR in the EVE lists than in the AM and PM lists. Patients with diabetes were preferentially listed for AM or PM procedures rather than EVE procedures. This likely resulted in a lower proportion of patients older than 60 years in the EVE list compared with the AM and PM
Table 3 a Univariate logistic regression analysis of factors associated with adenoma detection after exclusion of Bowel Cancer Screening Programme cases.

\begin{tabular}{|llll|}
\hline Variable & Odds ratio & $\mathbf{9 5} \% \mathbf{C l}$ & Pvalue \\
\hline Time & & & \\
\hline PM vs. AM & 1.08 & $0.81-1.44$ & 0.588 \\
\hline EVE vs. AM & 0.58 & $0.34-0.97$ & 0.038 \\
\hline EVE vs. PM & 0.53 & $0.31-0.93$ & 0.026 \\
\hline Age $>$ 60 vs. $\leq 60$ y & 2.67 & $2.23-3.17$ & 0.000 \\
\hline Queue position ${ }^{1}$ & & & \\
\hline 2 vs. 1 & 1.18 & $0.88-1.56$ & 0.263 \\
\hline 3 vs. 1 & 1.04 & $0.72-1.51$ & 0.830 \\
\hline 4 vs. 1 & 0.97 & $0.66-1.42$ & 0.876 \\
\hline 3 vs. 2 & 0.86 & $0.68-1.15$ & 0.369 \\
\hline 4 vs. 2 & 0.82 & $0.62-1.10$ & 0.194 \\
\hline 4 vs. 3 & 0.93 & $0.71-1.22$ & 0.612 \\
\hline Gender: female vs. male & 0.56 & $0.44-0.73$ & 0.000 \\
\hline Trainee presence & 1.02 & $0.69-1.52$ & 0.901 \\
\hline Bowel preparation & & & \\
\hline Satisfactory vs. poor & 1.75 & $1.15-2.66$ & 0.009 \\
\hline Good vs. poor & 1.82 & $1.18-2.81$ & 0.007 \\
\hline Satisfactory vs. good & 1.04 & $0.85-1.27$ & 0.700 \\
\hline Experience & 1.14 & $0.78-1.68$ & 0.492 \\
\hline
\end{tabular}

$\mathrm{Cl}$, confidence interval; $\mathrm{PM}$, afternoon; AM, morning; EVE, evening.

${ }^{1}$ Queue variable was divided into four categories: the fourth category consisted of queues $\geq 4$; age was divided into two groups (with 60 years used as a cutoff point) for data-fitting purposes.

Table 3b Multivariate logistic regression model of adenoma detection after exclusion of Bowel Cancer Screening Programme cases $(n=2058)$.

\begin{tabular}{|llll|}
\hline Variable & Odds ratio & $\mathbf{9 5 \% C l}$ & Pvalue \\
\hline Gender & 0.59 & $0.45-0.76$ & $<0.001$ \\
\hline Age group & 2.43 & $1.42-3.75$ & 0.001 \\
\hline $\begin{array}{l}\text { Bowel preparation: } \\
\text { satisfactory vs. poor }\end{array}$ & 2.31 & $1.31-3.44$ & 0.002 \\
\hline Time: EVEvs. AM + PM & 1.40 & $0.60-3.27$ & 0.432 \\
\hline Bowel preparation * time & 0.44 & $0.22-0.89$ & 0.021 \\
\hline
\end{tabular}

$\mathrm{Cl}$, confidence interval; EVE, evening; AM, morning; PM, afternoon.

lists. This difference was still present after the exclusion of BCSP cases. Thus, differences in case mix could have accounted for the lower ADR in the EVE lists.

Several recent studies have linked operator fatigue to a lower ADR. Surrogate markers of endoscopy fatigue that may influence the ADR include AM versus PM colonoscopy (intersession variation), increasing number of procedures within a session (intrasession variation), and finally the queue position as determined by the number of procedures before the index colonoscopy. Some studies have looked at both variations between sessions and variations in polyp yield within a session, whereas others have reported just on intersession variations in the ADR. For instance, Chan et al. reported a higher ADR in patients undergoing early morning procedures than in later cases after controlling for multiple factors in a university-based Veterans Affairs medical center [2]. They also reported a steady decline in ADR with each passing hour of the day. Similar findings have been reported by Sanaka et al. [4]. A third study also reported similar findings, but with a further observation of a decline in ADR with increasing queue position [7], which was subsequently replicated by the same authors [18]. However, this phenomenon of a lower ADR with increasing time of day was not observed by Lurix et al. [6] in their study of 3085 colonoscopies. They found similar ADRs in 
AM and PM sessions regardless of whether the endoscopist worked a full-day or half-day block [6]. In our study, there was no difference between the ADRs of AM and PM lists when they were compared as a whole. Furthermore, we observed that increasing queue position within each session did not adversely affect ADR.

Both the CIR and the ADR were significantly higher in the BCSP group than in an unselected cohort in our study. This finding is similar to the findings reported by a recent study [9]. The higher CIR is likely due to the fact that endoscopists in the BCSP are highly experienced at the time of accreditation for the BCSP. Patients in the BCSP represent an average-risk cohort in whom a higher ADR is expected. Both the CIR and the ADR in the BCSP cohort are in keeping with figures published in a recent study of the first million BCSP cases [19].

Trainee involvement did not influence the ADR in our study. At least two previous studies reported an increase in the ADR when a fellow was involved in the colonoscopy [20,21]. Interestingly, the increase in ADR was reported only when senior trainees were involved in the procedure. Because of the large number of trainees in our study and their varying levels of training, we were unable to test the association between level of training and ADR.

The quality of colon cleansing is a major determinant of adenoma detection and the technical success of colonoscopy [22]. The quality of bowel preparation varied in our study, with a higher proportion of patients in the AM and PM lists than of patients in the EVE lists having poor bowel preparation. This finding is consistent with those of recent studies suggesting that bowel preparation on the day of colonoscopy is superior to split-dose bowel preparation [23-25]. The adverse effects of poor bowel preparation on key indicators of colonoscopy performance reported in our study are consistent with previous observations [22].

Our study has some limitations. Firstly, the colonoscopy withdrawal time is not routinely recorded in our institute and was therefore not available for analysis. It is well recognized that a withdrawal time of less than 6 to 8 minutes is associated with a lower ADR $[26,27]$. It is likely that a reduction in withdrawal times among operators as the day progressed may have resulted in a lower ADR, but these data were not available. The start and end times of procedures were also not routinely recorded by the operator. Thus, we were unable to analyze whether the ADR declined with each progressive hour of the day; queue position was used as a surrogate instead. We included patients who underwent colonoscopy for any indication, as opposed to previous studies, which included only patients with an average risk for colon cancer. Our heterogeneous case mix may have resulted in variable ADRs across the lists. However, the results of the study were not influenced by the exclusion of patients at average risk for colon cancer. Finally, we did not record other clinically important variables, such as smoking status and BMI, which may have influenced the findings of our study.

In conclusion, with the rising workloads of endoscopists, it is important to monitor the effects of physician fatigue on the indicators of endoscopy performance. Reassuringly, our study found that colonoscopy performance remained stable throughout an extended working day when the procedures were done in halfday blocks. This information is important for administrators as they plan for the provision of colonoscopies by increasing the number of working sessions without compromising quality.

\section{Acknowledgments}

\section{$\nabla$}

The authors would like to acknowledge the contribution of the medical students who helped with data collection.

\section{References}

1 Bowel Cancer UK. Endoscopy in the UK. Available from: http://www. bowelcanceruk.org.uk/media/173462/1061_bcuk_endoscopy_report. pdf. Accessed May 21, 20152015

2 Chan MY, Cohen H, Spiegel BM. Fewer polyps detected by colonoscopy as the day progresses at a Veteran's Administration teaching hospital. Clin Gastroenterol Hepatol 2009; 7: 1217-1223 quiz 1143

3 Sanaka MR, Shah N, Mullen KD et al. Afternoon colonoscopies have higher failure rates than morning colonoscopies. Am J Gastroenterol 2006; 101: 2726-2730

4 Sanaka MR, Deepinder F, Thota PN et al. Adenomas are detected more often in morning than in afternoon colonoscopy. Am J Gastroenterol 2009; 104: 1659-1664 quiz 1665

5 Harewood GC, Chrysostomou K, Himy N et al. Impact of operator fatigue on endoscopy performance: implications for procedure scheduling. Dig Dis Sci 2009; 54: 1656 - 1661

6 Lurix E, Hernandez AV, Thoma $M$ et al. Adenoma detection rate is not influenced by full-day blocks, time, or modified queue position. Gastrointest Endosc 2012; 75: 827-834

7 Lee A, Iskander JM, Gupta $N$ et al. Queue position in the endoscopic schedule impacts effectiveness of colonoscopy. Am J Gastroenterol 2011; 106: 1457-1465

8 Munson GW, Harewood GC, Francis DL. Time of day variation in polyp detection rate for colonoscopies performed on a 3-hour shift schedule. Gastrointest Endosc 2011; 73: 467-475

9 Thurtle D, Pullinger M, Tsigarides J et al. Colonoscopic polyp detection rate is stable throughout the workday including evening colonoscopy sessions. F1000Res 2014; 3: 107

10 Ponchon T. ESGE/ESDO Quality in endoscopy: colonoscopy and colonic neoplasms symposium reports. Endoscopy 2012; 44: 1065-1066

11 Valori R. Joint Advisory Group on GI Endoscopy. BSG quality and safety indicators for endoscopy. http://www.thejag.org.uk/downloads\%5CUnit\%20Resources\%5CBSG\%20Quality\%20and\%20Safety\%20Indicators. pdf Updated March 2007. Accessed May 21, 2015

12 Gavin DR, Valori RM, Anderson JT et al. The national colonoscopy audit: a nationwide assessment of the quality and safety of colonoscopy in the UK. Gut 2013; 62: 242-249

13 Aslinia F, Uradomo L, Steele A et al. Quality assessment of colonoscopic cecal intubation: an analysis of 6 years of continuous practice at a university hospital. Am J Gastroenterol 2006; 101: 721 - 731

14 Shah HA, Paszat LF, Saskin $R$ et al. Factors associated with incomplete colonoscopy: a population-based study. Gastroenterology 2007; 132: $2297-2303$

15 Gurudu SR, Ratuapli SK, Leighton JA et al. Adenoma detection rate is not influenced by the timing of colonoscopy when performed in half-day blocks. Am J Gastroenterol 2011; 106: 1466 - 1471

16 Rex DK. Maximizing detection of adenomas and cancers during colonoscopy. Am J Gastroenterol 2006; 101: 2866 - 2877

17 Rex DK, Bond JH, Winawer S et al. Quality in the technical performance of colonoscopy and the continuous quality improvement process for colonoscopy: recommendations of the U. S. Multi-Society Task Force on Colorectal Cancer. Am J Gastroenterol 2002; 97: 1296-1308

18 Lee TJ, Rees CJ, Blanks RG et al. Colonoscopic factors associated with adenoma detection in a national colorectal cancer screening program. Endoscopy 2014; 46: $203-211$

19 Lee TJ, Rutter MD, Blanks RG et al. Colonoscopy quality measures: experience from the NHS Bowel Cancer Screening Programme. Gut 2012; 61: 1050 - 1057

20 Peters SL, Hasan AG, Jacobson NB et al. Level of fellowship training increases adenoma detection rates. Clin Gastroenterol Hepatol 2010; 8: 439-442

21 Rogart JN, Siddiqui UD, Jamidar PA et al. Fellow involvement may increase adenoma detection rates during colonoscopy. Am J Gastroenterol 2008; 103: $2841-2846$

22 Froehlich $F$, Wietlisbach $V$, Gonvers JJ et al. Impact of colonic cleansing on quality and diagnostic yield of colonoscopy: the European Panel of Appropriateness of Gastrointestinal Endoscopy European multicenter study. Gastrointest Endosc 2005; 61: $378-384$ 
23 Chiu HM, Lin JT, Wang HP et al. The impact of colon preparation timing on colonoscopic detection of colorectal neoplasms-a prospective endoscopist-blinded randomized trial. Am J Gastroenterol 2006; 101: 2719-2725

24 Parra-Blanco A, Nicolas-Perez D, Gimeno-Garcia A et al. The timing of bowel preparation before colonoscopy determines the quality of cleansing, and is a significant factor contributing to the detection of flat lesions: a randomized study. World J Gastroenterol 2006; 12 : $6161-6166$
25 Varughese S, Kumar AR, George A et al. Morning-only one-gallon polyethylene glycol improves bowel cleansing for afternoon colonoscopies: a randomized endoscopist-blinded prospective study. Am J Gastroenterol 2010; 105: $2368-2374$

26 Barclay RL, Vicari IJ, Doughty AS et al. Colonoscopic withdrawal times and adenoma detection during screening colonoscopy. N Engl J Med 2006; 355: 2533-2541

27 Barclay RL, Vicari JJ, Greenlaw RL. Effect of a time-dependent colonoscopic withdrawal protocol on adenoma detection during screening colonoscopy. Clin Gastroenterol Hepatol 2008; 6: 1091 -1098 